\title{
All-optical switching and real-time spectroscopy of soliton molecules in a few-cycle laser oscillator
}

\author{
Felix Kurtz ${ }^{1}$, Daniel Solli ${ }^{1,2}$, Bahram Jalali ${ }^{2}$, Claus Ropers ${ }^{1}$, and Georg Herink ${ }^{3}$ \\ ${ }^{1}$ IV. Physical Institute, University of Göttingen, Germany \\ ${ }^{2}$ Department of Electrical Engineering, University of California Los Angeles, USA \\ ${ }^{3}$ Institute of Physics, University of Bayreuth, Germany
}

\begin{abstract}
Bound states of femtosecond solitons are generated and controlled in a commercial sub-10 fs Kerr-lens mode-locked ultrashort oscillator. Using real-time time-stretch interferometry, we resolve the resonance of vibrating soliton molecules and demonstrate all-optical switching between stable bound-states of different binding distance.
\end{abstract}

\section{Introduction}

Interactions between individual solitons are observed in a variety of physical systems, spanning from optical fibers, photorefractive media, water surfaces to BoseEinstein condensates.

Mode-locked lasers represent dissipative nonlinear systems which routinely generate single solitonic pulses but - more generally - are known to support complex bound states of multiple solitons [1,2]. Rapid dynamic interactions between soliton complexes with pico- to femtosecond separations are typically inaccessible to standard laser characterization techniques, such as scanning interferometric autocorrelation, temporally-averaging spectroscopy or single-shot nonlinear detection.

Here, we present real-time access to rapid bound state dynamics in a commercial few-cycle broadband Kerr-lens mode-locked Ti:sapphire oscillator. Employing a frequency-dependent external perturbation we probe the resonance behaviour of optical soliton molecules which parallels molecular oscillators. Furthermore, we implement all-optical control of the soliton molecule states and demonstrate the reversible switching of femtosecond soliton separations. The presented scheme presents a unique testbed for the complex dynamics of dissipative soliton bound states and external control strategies thereof. 


\section{Real-Time Spectral Interferometry}

Time-stretch dispersive Fourier transform (TS-DFT) and high-speed real-time sampling enables the observation of rapid intracavity soliton dynamics with femtosecond separations at the single-shot level and over hundreds of thousand consecutive roundtrips $[3,4]$. This allows for the mapping of transient and periodic interactions between multiple closely-spaced solitons normally hidden from view. The optical spectra of soliton complexes correspond in the frequency domain to spectral interferograms, encoding both the separation and the relative phase of its constituents, see Figure 1. In order to access this information for fast bound-state dynamics, rapid spectral detection is essential [5-9]. The demonstrated single-shot spectroscopy is enabled via the transformation of spectral information to the timedomain, and subsequent high-bandwidth real-time acquisition.

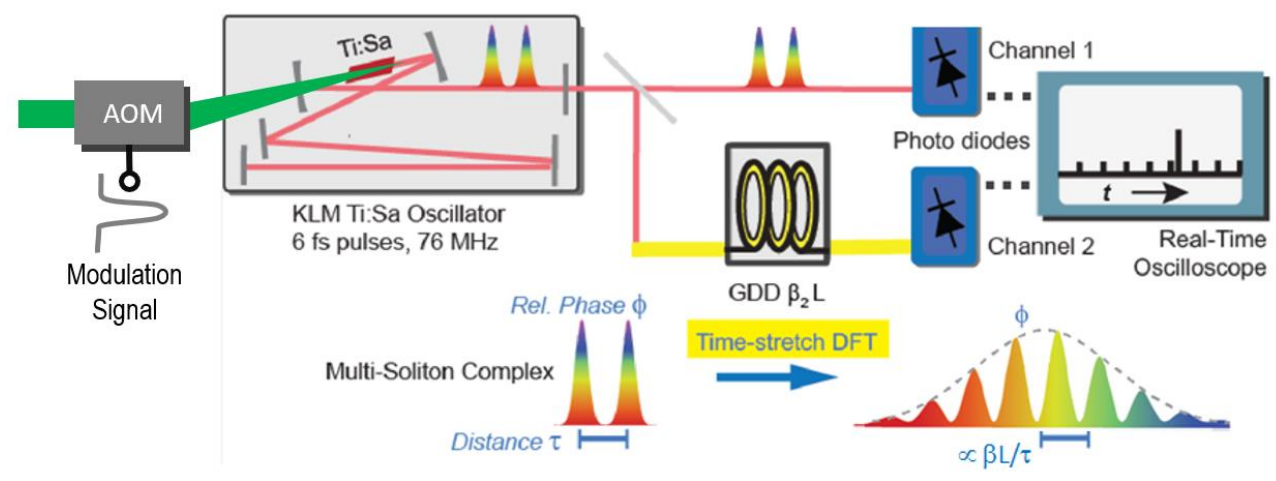

Fig. 1 Real-time detection of transient soliton molecules via TS-DFT and spectral interferometry: Soliton separations and relative phases are recorded via time-stretch spectral interferometry. Active control of soliton dynamics is achieved via rapid modulations of the pump power facilitated by acousto-optic beam deflection.

\section{Resonant Soliton Molecule Excitation}

In analogy to molecular oscillators, the effective binding potential of a soliton molecule allows for resonant excitations of soliton vibrations with respect to their separation and relative phase. We employ acousto-optic amplitude modulation of the pump power as a rapid external control parameter. Sweeping the pump modulation frequency over time and recording the soliton vibration in real-time, enables us to to map the resonance behaviour of the soliton interaction. We observe a Fano-type response of the dynamic bound state which is related to the interplay of Kerrnonlinearity, dispersion and gain saturation. The resultant tuning curves present excitation spectra of the soliton bound state and corroborate the notion of such bound states as soliton "molecules" with characteristic resonance properties.

\section{All-optical Switching of Soliton Molecules}

Rapid external control of the dissipative system via the pump-power allows for tailored perturbations of the dissipative system on $\mu$ s-timescales. Employing transient 
reductions in the pump power (Fig. 2c), we demonstrate the switching between different states of stable soliton molecules with locked relative separation and phase, as shown in the real-time interferograms in Figure 2a. The respective Fouriertransform is displayed in Figure $2 \mathrm{~b}$ and reveals the evolution in the temporal pulse separation during pump power modulation (Fig. 2c). In particular, we can reversibly switch between bound states of different separations. These results demonstrate the possibility of all-optical control of femtosecond bound states, bearing novel directions for ultrafast metrology schemes and for the realization of all-optical memory.

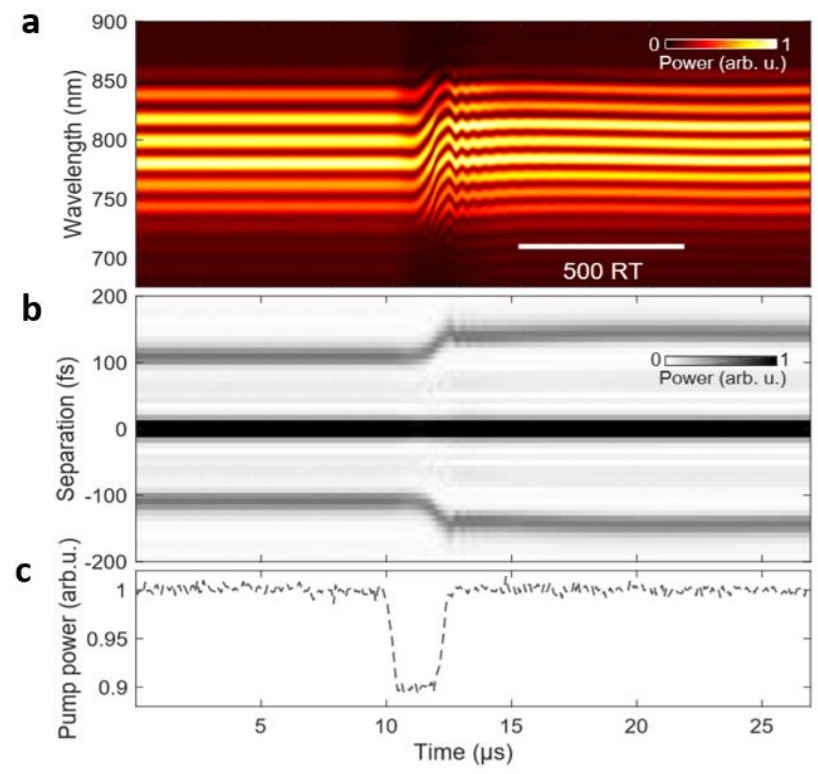

Fig. 2. (a) Experimental consecutive real-time interferograms recorded continuously during a microsecond external modulation of the laser pump power. (b) The respective Fourier-transforms indicate the field autocorrelation and the rapid switching between two distinct bound states of stable separation. (c) The external control is facilitated via the temporal modulation of the pump power via acousto-optic deflection.

\section{References}

[1] P. Grelu, N. Akhmediev, Nat. Photon. 6, 84-92 (2012).

[2] M. Lederer, M. et al., JOSA B 16 (6), 895-904 (1999).

[3] P. Kelkar, F. Coppinger, A. Bhushan, B. Jalali, Electronics Letters 35, 1661-1662 (1999).

[4] D. Solli, G. Herink, B. Jalali, C. Ropers, Nat. Photon. 6, 463-468 (2012).

[5] P. DeVore, et al., IEEE Photonics Journal 6 (2014).

[6] S. Sugavanam, et al., presented at CLEO 2015.

[7] G. Herink, B. Jalali, C. Ropers, D.R. Solli, Nat. Photon. 10, 321-326 (2016).

[8] K. Krupa, K. Nithyanandan, U. Andral, P. Tchofo-Dinda, and P. Grelu, Phys. Rev. Lett. 118, (2017).

[9] G. Herink, F. Kurtz, B. Jalali, D.R. Solli, C. Ropers, Science 356, 50-54 (2017). 